\title{
Postburn dorsal and palmar interdigital commissural contractures: Anatomy and treatment-A new approach
}

\author{
Viktor M. Grishkevich \\ Department of Reconstructive and Plastic Surgery, A.V. Vishnevsky Institute of Surgery of the Russian Academy of Medical Sci- \\ ences, Moscow, Russia \\ Email: grishkevichmail@gmail.com
}

Received 19 January 2013; revised 1 March 2013; accepted 7 April 2013

Copyright (C 2013 Viktor M. Grishkevich. This is an open access article distributed under the Creative Commons Attribution License, which permits unrestricted use, distribution, and reproduction in any medium, provided the original work is properly cited.

\begin{abstract}
Background: Postburn dorsal and palmar interdigital commissural contracture is one of the most common complications of hand burns which restricts finger motion and presents a serious cosmetic defect. Many techniques and flaps have been suggested, and research continues for more effective techniques as the problem has not been solved. Methods: Anatomy of scar interdigital commissural contractures was studied in 760 operated patients aiming to develop a new, more effective surgical technique. Results: There are two anatomic types of scar commissural contractures: edge and total. Dorsal and palmar commissural contractures are identified as the edge type and are caused by the fold located along interdigital fossa's edge. In the fold, the lateral sheet is scars, and the medial sheet and adjacent interdigital fossa are healthy skin. Total commissural contracture is characterized by interdigital fossa obliteration and phalanges' fusion. Scar sheets have a trapeze-shaped surface deficiency (contracture cause), which spreads from the fold's crest to metacarpophalangeal joints. The surgical technique consists of scar surface deficiency compensation and commissural groove and slant restoration which can be optimally achieved with the trapezoid adipose-cutaneous flap. The best donor site is interdigital fossa. The small skin grafts on the lateral surface of proximal phalanges did not present a cosmetic defect. Conclusion: Dorsal and palmar interdigital commissural contractures are of an edge type and can be successfully reconstructed with trapezeflap plasty.
\end{abstract}

Keywords: Postburn Syndactyly; Syndactyly Treatment; Hand Burn Complications; Trapezoid Flap; Trapeze-Flap Plasty

\section{INTRODUCTION}

One of the frequent consequence of hand burns is the interphalangeal scar commissural contracture that is characterized by the fold formation along the edge of interdigital fossa, smoothing and deforming the commissural groove and slant. Finger motion restriction and significant cosmetic defect are an indication for surgery. Multiple reconstructive options have been suggested for interdigital space restoration [1,2], such as skin grafting and various local flaps. An optimal technique, however, that would lead to perfect functional and good cosmetic results is yet to be explored. Reconstruction should not only release the contracture but also restore all commissural components: interdigital fossa's depth and form, interphalangeal groove and slant up to metacarpal head level. Our vast experience in this field showed that most commonly used current procedures are unable to release the contracture fully while simultaneously restoring interphalangeal groove and slant on all its extent, which would prevent the contracture recurrence and yield good cosmetic outcomes. Dissatisfied with results, we studied the commissural contracture more carefully considering the possibility of using a flap from interdigital fossa for contracture elimination and all commissural components' restoration. The achieved satisfying results prompted the development of a new approach to surgical treatment presented in this paper.

\section{MATERIAL AND METHODS}

760 patients with postburn interdigital commissural contractures, 420 men and 340 women, from 5 to 65 years old, were operated personally; 76 of them were children; 635 of patients had first web space contractures that were eliminated simultaneously. Every patient had 1 to 6 contractures on one or both hands. Dorsal commissural contracture was diagnosed in 623 patients (82\% of the total 
number); palmar, in 84 patients (11\%), and total contracture, in 53 patients (7\%). Rough, thick scars on hand's dorsal surface in 65 patients were excised, and the wound was resurfaced with split skin grafts simultaneously with commissural groove and slant restoration with a trapezoid flap. In half of the patients, palmar commissural contracture was combined with palm contracture and finger flexion contracture; all contractures of the hand palmar surfaces were eliminated simultaneously using specific reconstructive techniques. Before and during the operation, attention was paid to the anatomic features of contractures: scar location and spreading on the dorsal and palmar hand surfaces and fingers, the fold's presence and its location in relation to interdigital fossa, the fold's features (contracture type). Moreover, the interdigital fossa's depth, interdigital grove, and slant were observed in finger's abduction position; metacarpophalangeal joint flexion and extension were also observed. Immediate and follow-up (from 6 months to 15 years) results were evaluated using the following criteria: 1) Presence of residual or recurred contracture; 2) Full or partial restoration of the following components: a) interdigital fossa's depth and form, b) interphalangeal groove, and c) slant on all its normal extension, starting from the metacarpal bone heads’ level; 3) Donor site morbidity.

The results were considered positive if the anatomy of commissures was fully restored in comparison with healthy hand, in which, after fingers' abduction (dorsal hand view), the interdigital commissural space accepts a form of a groove, slanting smoothly from metacarpal bone heads level to the palmar interdigital fold. In finger abduction, the space among proximal phalanges is easily increased due to skin's surplus and its elasticity. Skin excess in interphalangeal groove allows for fingers to diverge and have separate motions in metacarpophalangeal joints. When the hand is in a rest (comfort) position, approximately half of the groove is smoothed. When metacarpophalangeal joints flex, a fossa appears between the heads of the metacarpal bone heads. Therefore, the interdigital commissure, as a separate hand unit, should be estimated in fingers' abduction and adduction position, in metacrpophalangeal joints extension and flexion. Therefore, the commissural contracture treatment is aimed at restoring its anatomic components: the commissural groove, slant, intermetacarpal heads' fossa and interdigital fossa's shape and depth. These normal anatomic characteristics were used as criteria for evaluation of the effectiveness of the surgical techniques.

\section{RESULTS}

\subsection{Anatomy of Commissural Contractures}

Based on anatomic features, the commissural contractures were classified into two types: edge (dorsal and pal- mar), making up $93 \%$ of the total number, and total (7\%).

\subsubsection{Anatomy of Dorsal Commissural Contracture (Figure 1)}

Dorsal commissural contractures, making up $82 \%$ of the total number, are the result of dorsal hand surface burns. At time of burns, fingers adduct; therefore, half of the proximal interphalangeal space (groove and slant) is injured with the dorsal hand at the same degree. While the wound is healing, the fingers are in an adduction position; therefore, contracted inelastic scars cover nearly half of the interphalangeal space, smoothing and deforming the groove and slant (Figure 1(a)), forming a crescent-shaped fold along the dorsal edge of interdigital fossa (Figures 1(a) and (b)). The fossa between the metacarpal bone heads also becomes smoothed. The fold, as a hood, covers the proximal half of the interdigital space (fossa) partially or fully. The lateral (dorsal) sheet (in relation to interdigital fossa) of the fold is scars and is the cause of the contracture due to surface deficiency (Figure 1(a)). The contracted scar's surface deficiency extends from the fold's crest (where it is maximal) to the metacarpal bone heads and has a trapezoid form (Figures 1(c) and 2(c)). The medial fold's sheet is the healthy skin which spreads over the interdigital undamaged fossa (Figure 1(a), lower, B). The fold's crest is the edge of scars (Figures 1(a) and (b)). Thus, the dorsal commissural scar contracture is identified as the edge type, having three specific anatomic characteristics: 1) the fold is formed along the interdigital fossa edge; 2) the crest of the fold is the edge of scars; 3) therefore, the crescent-shaped fold consists of two distinctively different sheets: scar lateral (according to the interdigital fossa) and healthy skin medial sheet. These three anatomic features characterize the edge scar contractures regardless of their localization [3, 4].

Commissural edge contracture is caused by the scar sheet surface deficiency which is necessary to determine as the basis for reconstruction: reconstruction involves scar surface deficiency compensation with a flap of a similar shape. The scar surface deficiency is measured as follows: lateral (dorsal) scar sheet of the fold is separated from the medial healthy sheet with an incision along the fold's crest; then, the contracture is released with the scar sheet dissection from the fold's crest to metacarpal bone heads, or metacarpophalangeal joints' rotation axis with a Y-shaped incision. The Y-shaped incision (not going beyond the rotation axis level) is necessary for complete contraction release. After fingers' abduction, the wound, as a rule, accepts a trapezoid form, reflecting the size and form of scar surface deficiency (Figure 1(c)) regardless of contracture severity (Figure 2(c)). This fact, that scar surface deficiency (real cause of contracture, groove and 


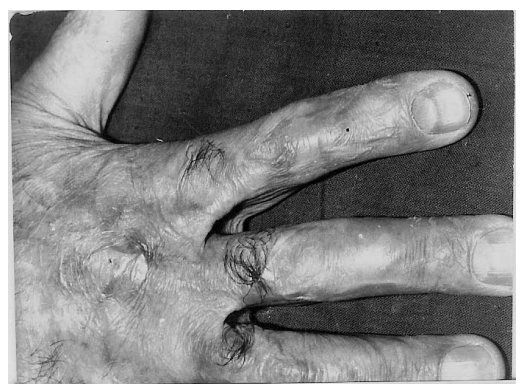

(a)

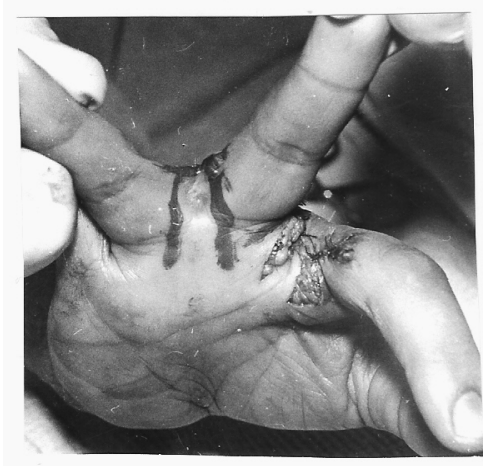

(b)
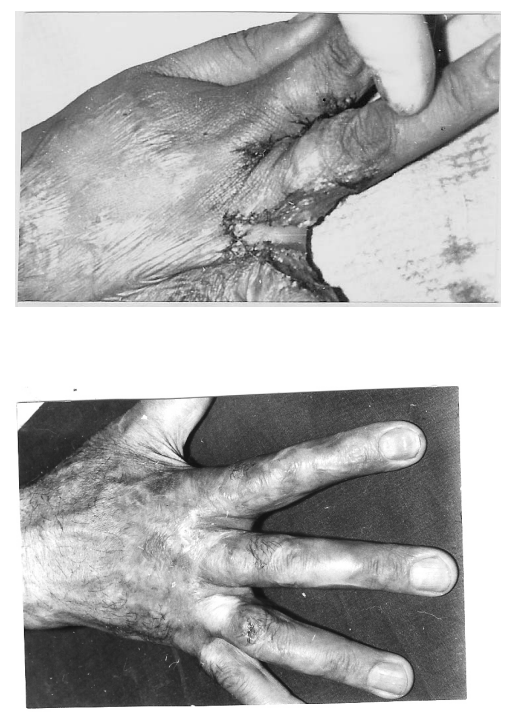

(e)

(d)
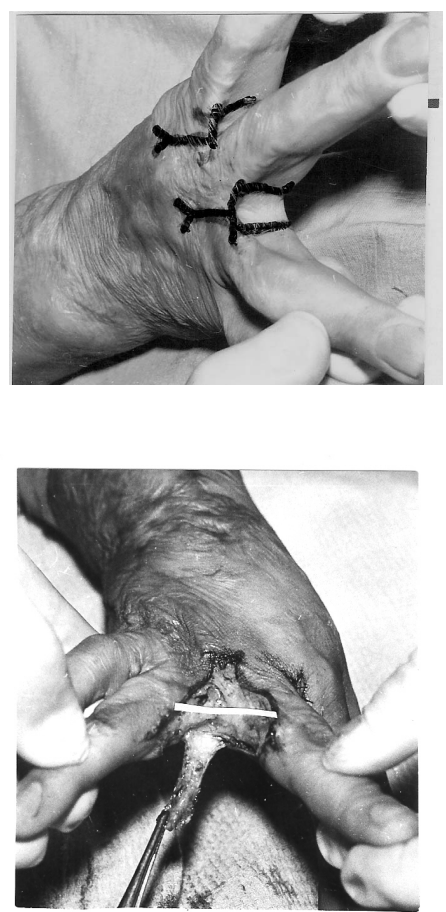

(c)
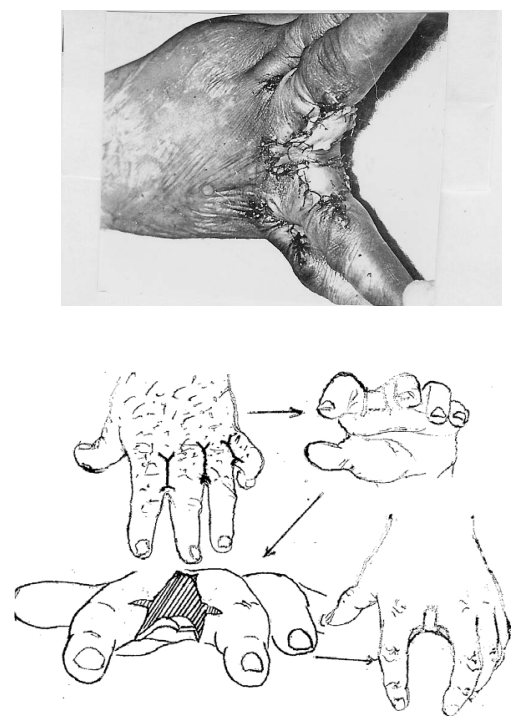

(f)

Figure 1. Anatomy of postburn dorsal interdigital contracture (syndactyly) and reconstruction with trapeze-flap plasty. (a) Upper-dorsal hand scars smoothed commissural groove and scant, formed fold along dorsal edge of interdigital fossa; in the fold: lateral (dorsal) sheet is scars; (below)-medial sheet and fossa is healthy skin; planning of operation ((a) and (b)): Y-shaped line for contracture release from fold's crest to the metacarpal bone heads; line along fold's crest for sheets separation; two lines in interdigital fossa are flap's contours; palmar interphalangeal fold and $2 \mathrm{~mm}$ of palm is included in the flap; (c) After scar sheet dissection and finger abduction, the wound (scar surface deficiency) accepted trapezoid form (above the white strip; lower, donor wound), trapezoid adipose-cutaneous flap mobilized; scar surface deficiency (cause of contracture) spreads from the fold's crest to the metacarpal bone heads; (d) Upper- flap advanced on the wound and sutured with dissected scar's wound forming the groove and slant; wounds on the lateral surfaces of proximal phalanges; (d) Lower small donor wound on inner surface of proximal phalanges split skin grafted; (e) Follow-up result: contracture fully eliminated; commissural groove, slant and fossa's depth restored; F-scheme of operation. 


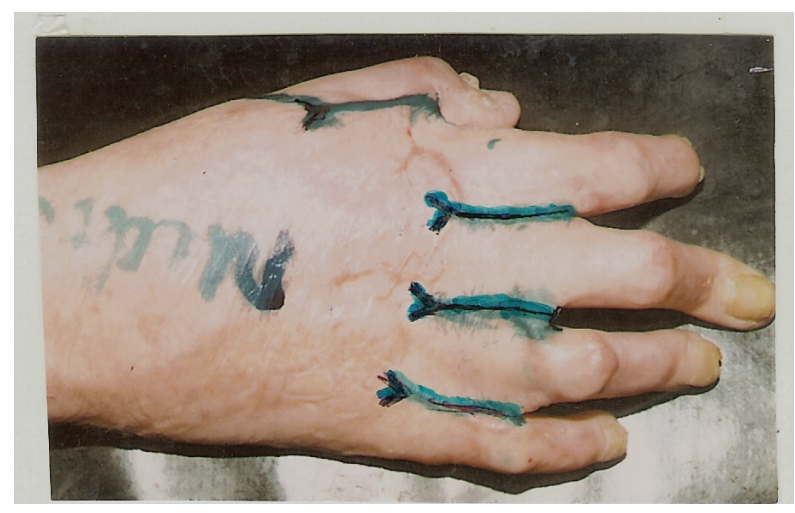

(a)

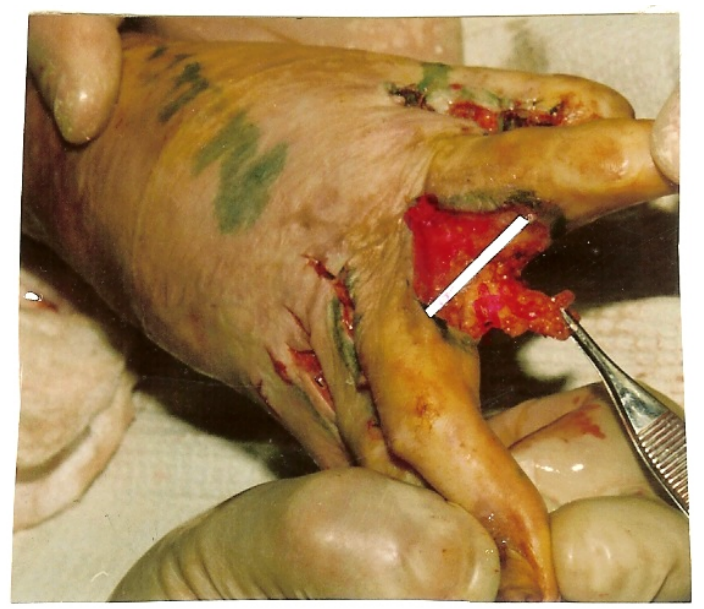

(c)

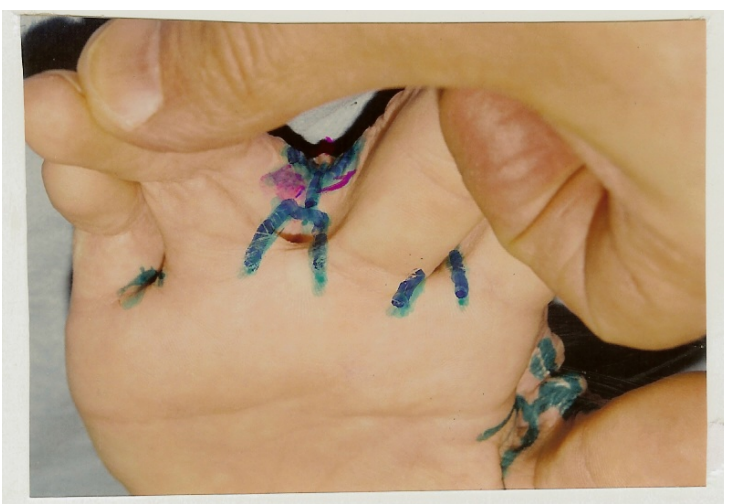

(b)

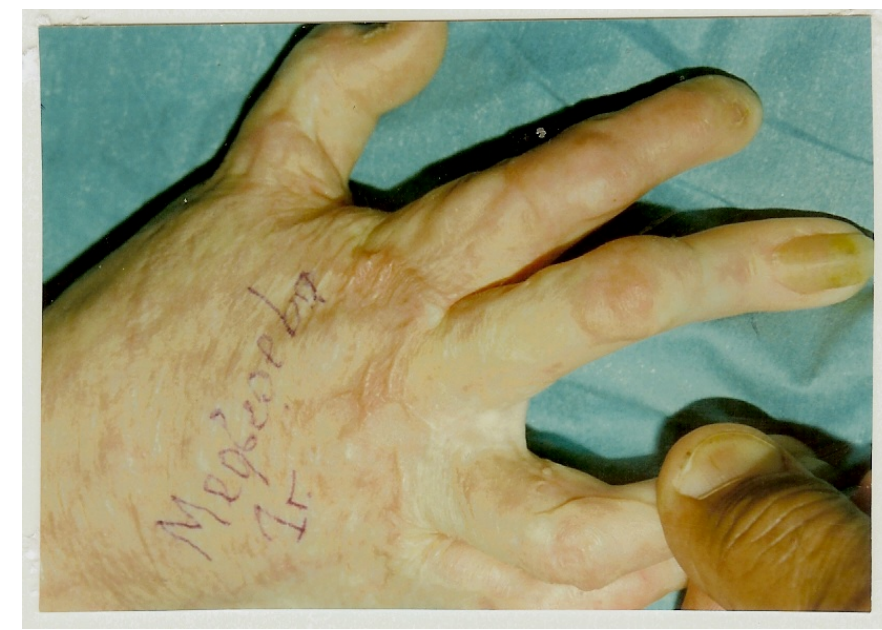

(d)

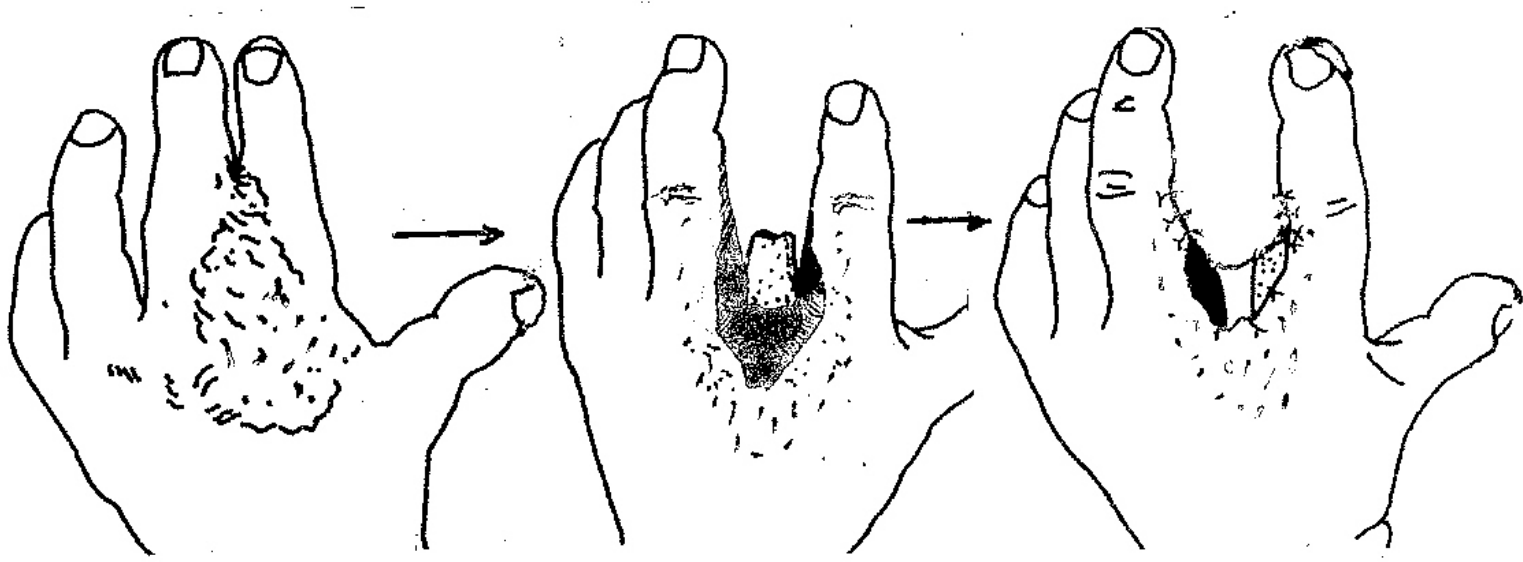

(e)

Figure 2. Technique for severe dorsal commissural contracture treatment and result. (a) Before operation: grade IV dorsal contracture, Y-shaped line for contracted scars' dissection from fold's crest to the metacarpal bone heads; (b) Volar surface: line for scars dissection between proximal phalanges from the fold's crest to notch, proximal of which the flap's contours are outlined; (c) Contracted scars dissected, flap mobilized, white strip divided wound on upper-trapezoid scar surface deficiency, and lower-donor wound; (d) Result of reconstruction (one year after operation): contracture removed, commissural groove, slant and fossa' depth fully restored; (e) Scheme of reconstruction.

slant deformity) has a trapezoid form, is a key for understanding the selection process for flap's form needed for adequate burned commissure restoration. The form/shape of the scar surface deficiency, which is a trapeze, is used as grounds for development of the new approach and technique for most effective commissure resurfacing. Thus 
it became clear that the most suitable reconstructive technique for contracture release, interphalangeal groove and slant restoration should be based on use of the flap of a similar form (trapezoid) (Figures 1(b) and 2(b)). This further explains why triangular local flap plasty (Y$\mathrm{V}$ plasty and, Z-plasty and their modifications) are unable to adequately compensate scar surface deficit, fully release contractures, and restore the interphalangeal groove and slant.

Scars, covering the dorsal hand and fingers and forming the lateral fold sheet, can spread more distally, fusing dorsal surfaces of proximal phalanges (Figure 2(a)). Amidst these scars and healthy medial fold sheet lies the fossa (notch), visible from the palmar side (Figure 2(b)). These anatomic features pre-determine a different curative technique choice.

\subsubsection{Palmar Commissural Contracture Anatomy (Figure 3)}

Palmar commissural contracture is a result of hand palmar surface burns (Figures 3(a) and (b)). While the wound is healing and scarring, the existing palmar fold spreads in distal direction fusing the proximal phalanges and forming the palmar interdigital contracture. The fold consists of two sheets: the medial sheet, which is healthy skin that spreads on undamaged interdigital fossa, and the palmar (lateral) sheet (scars spreading over the palm). The contracture is caused by the palmar scar sheet's surface deficiency. After sheets' separation with an incision along the fold's crest and scar (palmar) sheet dissection with a Y-shaped incision to complete contracture release, and fingers abduction, the wound, as a rule, accepts a trapezoid form (Figure 3(c)). The trapeze-shaped wound reflects the size and form of the scar sheet deficiency. Consequently, for effective contracture elimination a similar to the wound's shape (trapezoid) flap is needed.

\subsubsection{A Total Commissural Contracture}

Is formed after severe circular burns hand including fingers and interdigital fossa. Wound healing results in interdigital fossa obliteration and fingers' lateral surface fusion. Severe scar surface deficiency excludes reconstruction with local and adjacent tissues.

\subsection{Surgical Technique}

Reconstruction consists of three parts: a) complete contracture release, b) flap mobilization and wound resurfacing with the flap, and c) donor wound covering using skin grafting. The success of reconstruction depends on the optimal donor site choice that would allow necessary flap raise with minimal deformity. Selection of the interdigital fossa for trapezoid flap elevation is a second component of the new approach. The techniques for dorsal and palmar commissural contracture treatment are dis- tinctively different.

\subsubsection{Dorsal Contracture Reconstruction (Figure 1)}

Planning lies in drawing of four lines in each contracted commissure: $1^{\text {st }}$-along the fold's crest; $2^{\text {nd }}$-a Y-shaped line perpendicular to the first one, passing through the center of the interphalangeal space from the fold's crest to the metacarpal bone heads; third and fourth lines outline trapezoid flap's borders in interdigital fossa (Figures 1(a) and (b)). The last lines end on the palm, $2 \mathrm{~mm}$ proximally to the palmar interdigital fold's crest (Figure 1(b)). The width of the flap's end should be by a $1 / 3^{\text {rd }}$ wider than the wound's width between metacarpal bone heads' level, and should equal 5 - $7 \mathrm{~mm}$ and should include a part of the fold's crest. The flap's base is wider than its end according to the wound's form. In all cases, the flap's width should exceed the wound by $1 / 3^{\text {rd }}$. The flap's length is approximately $25 \mathrm{~mm}$ and varies (as the width), depending on the measurement of the hand (adult vs. children). The length exceeds the width (middle parts of the flap) by 2 - 2.8 times. The specifics of the flap can be summarized as follows: the flap's end is wide, subcutaneous fat layer is included in the flap, supplying steady blood circulation, and the flap does not undergo rotation. Therefore, tissue necrosis and flap loss does not occur despite the flap's tension during its transposition on the wound; and the ratio value (length to width) has no practical meaning or application due to the aforementioned viability qualities of the flap.

Technical details (Figure 1). First, the fold's sheets are separated with an incision along the fold's crest; then, a Y-shaped incision, scars and subcutaneous fat layer from the fold's crest to the metacarpal bone heads. After fingers' abduction, the trapeze-shaped wound appears (Figures 1(c) and 2(c)). The wound reflects the form and size of the scar surface deficiency and suggests the flap to be further raised. According to the wound size and form (scar surface deficiency), but approximately $1 / 3^{\text {rd }}$ wider, a trapezoid flap is mobilized. The flap, which has a wide end and no acute angles, includes the skin and subcutaneous fat layer of interdigital fossa, supplying steady blood circulation despite the number of contractures on one hand (Figures 1 and 2). The flap's base includes the interdigital palmar fold and about $2 \mathrm{~mm}$ of palmar skin which makes the flap longer. After flap elevation, the interdigital commissure (fossa) is deepened, which shortens the wound's length (in anterio-posterior direction) and making the flap relatively longer. The raised flap shrinks and appears narrower than it really is and shown in photos; moreover, the flap, is advanced on the wound with tension and becomes longer and also thinner, and this is only its temporal appearance. The flap's end is connected with 2-3 sutures to the wound's edge at the level of metacarpal bone heads. Lateral flap's 


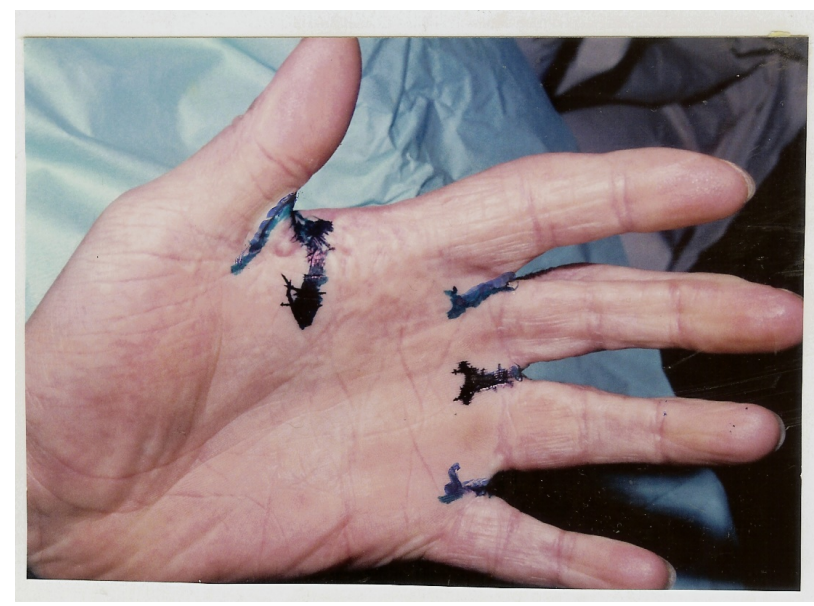

(a)

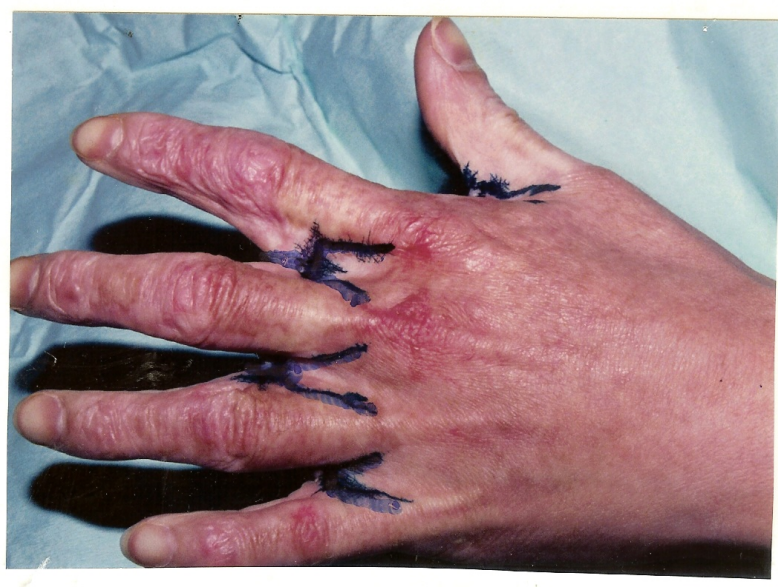

(b)

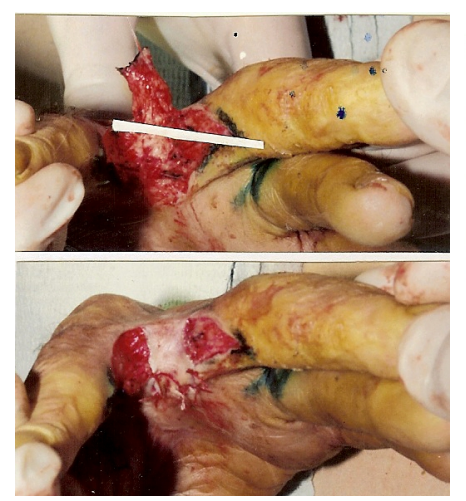

(c)

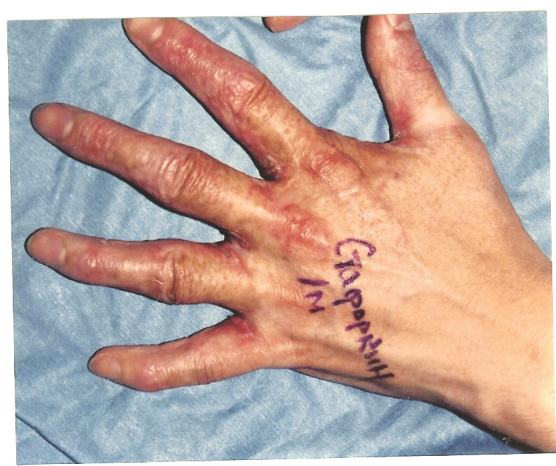

(d)

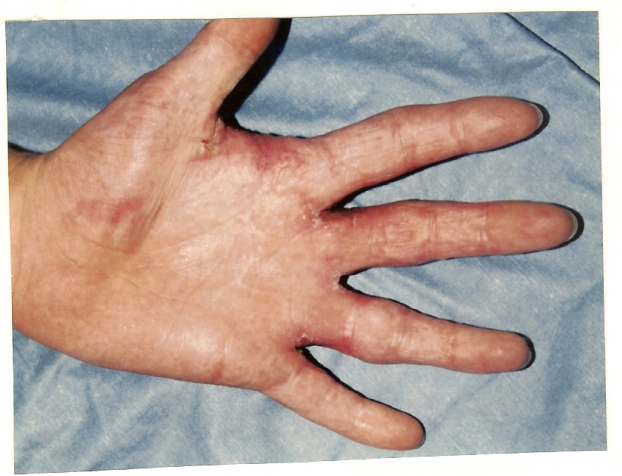

(e)

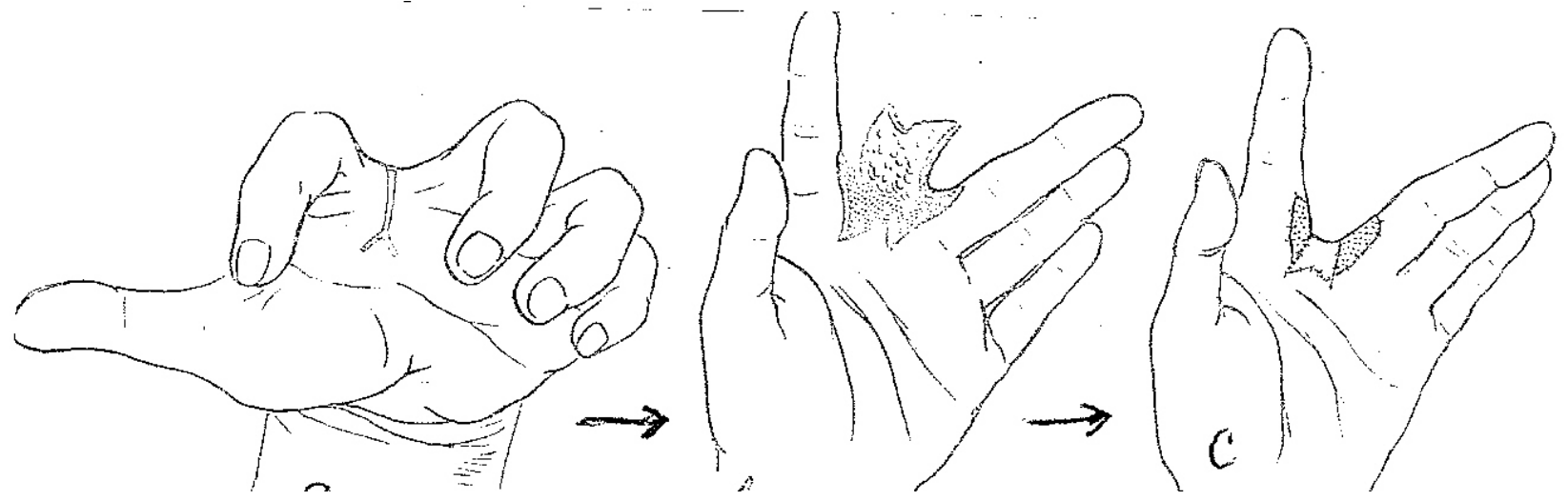

(f)

Figure 3. Technique for palmar interdigital commissural contracture treatment. (a) and (b) Contracture anatomy: interdigital commissural fold is located along palmar fossa's edge; palmar sheet of the fold is scars, dorsal sheet and fossa is healthy skin; planning of operation: lines for contracted scars dissection and flap's borders; (c) Upper-after scars' dissection the trapezoid wound is formed (lower of white strip); adipose-cutaneous flap elevated, donor wound-upper strip; (c) Lower-transposed flap compensated scar surface deficit, contracture eliminated; donor wound aside the flap will be skin grafted; (d) and (e) Result: contracture removed, all commissural components restored in full; (f) Scheme of operation.

borders are connected to the borders of dissected scar sheet (Figure 1(d), upper). Thick scar edges are thinned by an excision of inner scar layer up to 2 - $3 \mathrm{~mm}$ for better flap adaptation.

Due to interdigital fossa deepening and flap transposi- tion with tension, the small donor wounds (nearly $1 \mathrm{~cm}$ in diameter) are formed on the lateral surface of proximal phalangs (Figure 2(d), upper) which are covered with split skin grafts (Figure 2(d), lower). The transposed flap covers wound, compensates scar surface deficiency, 
and restores interdigital commissure, interphalangeal groove and slant (Figure 1(e)). The scheme of the operation is shown in Figure 1(f).

In case of severe contracture, the fused proximal phalanges are separated up to the medial healthy sheet where a small dimple is present (Figures 2(a) and (b)). Rough scars on the fingers are excised; thick scars are made thinner by excising inner scars' layers, and the wound of the proximal phalangs is primarily closed. After that, the commissure is reconstructed using the described above technique. After scar sheet dissection up to metacarpal heads with Y-shaped incision and finger abduction, the trapezoid wound appears (Figure 2(c), above white strip), reflecting scar surface deficiency. According to the wound's size and form, the adipose-cutaneous flap is elevated (Figures 2(b) and (c)). The transposed flap compensates scar surface deficit, eliminates the contracture, restores interdigital fossa, and interphalangeal groove and slant (Figure 2(d)). The scheme of the operation is shown in Figure 2(e).

The split skin transplant for proximal phalangs' donor wound covering is harvested from the inner shoulder's surface; the donor wound is primarily closed. All commissural contractures are reconstructed in one-stage surgery.

In case of total dorsal hand resurfacing, the commissural groove and slant are also restored with a similar adipose-cutaneous trapezoid flap (Figure 4). After scars' excision including the lateral fold sheet, the flap is elevated in interdigital fossa which is made deeper. The flap is transposed on a dorsal interphalangeal space; its end is fixed with two catgut sutures to the wound tissues between the metacarpal bone heads. The skin graft, taken from the lateral surface of hip joint, covers the hand dorsal surface; the wounds on the lateral surface of proximal phalangs should be preferably covered with separate pieces of skin graft.

\subsubsection{Palmar Contracture Reconstruction (Figure 3)}

Planning consists of drawing of four lines: along the fold's crest for separation of scars from healthy skin; Yshaped line indicating the level for contracted scars' dissection (middle length of proximal phalanges); the trapezoid adipose-cutaneous flap is planned in interdigital fossa with its base located in fossa's dorsal edge (Figures 3(a) and (b)).

Surgical details. First incision separates the scar lateral (palmar) fold sheet from medial healthy sheet. With a perpendicular Y-shaped incision, the contracture is released from the fold's crest to the metacarpal bones' heads. (Y-shaped incision is a necessary component ensuring easy scars' edges' divergence and complete contracture release). As a rule, after fingers' abduction, a trapezoid would is formed (Figure 3(c), upper, below the white strip), reflecting the size and form of scar surface deficiency. According to the trapeze-shaped wound's size, the adipose-cutaneous flap is raised in interdigital fossa (Figure 3(c), upper, above the white strip). For neurovascular bundle preservation, the incisions are directed parallel to the lateral surfaces of proximal phalangs. After fossa's deepening, the flap is transposed on the wound with tension, compensating scar sheet surface deficiency, eliminating contracture, and restoring interdigital commissure (Figure 3(c), lower). The wounds located asides the flap, on the lateral surfaces of proximal phalanges (Figure 3(c), lower) are split skin grafted. As a result, the contracture is fully released, commissural groove and slant are fully restored (Figures 3(d) and (e)). A scheme for this operation is shown in Figure 3(f).

After circular hand burns, dorsal or palmar commissural scar contractures can be combined with the finger flexion contractures. Both contractures are released simultaneously; the commissures are restored first.

\subsection{Results of Operations}

Dorsal and palmar edge commissural contractures were fully eliminated in all 760 patients using trapeze-flap plasty. A normal commissural groove and slant were achieved; fossa's depth and width were restored (Figures 1-5). No flap or its tip loss occurred regardless of width/ length flaps' ratio or quantity and severity of simultaneously reconstructed commissural contractures. Steady arterial and venous circulation prevented necrosis, and no other serious postoperative complications occurred influencing the final outcomes. Moreover, good blood supply allowed flaps' transposition on the dorsal hand with tension, posing no danger of tissue necrosis. Skin grafts on the inner surface of proximal phalanges grew well without complications. Grafts' shrinkage was insignificant and did not affect the initial postoperative depth of interdigital fossa (did not make it shallow); therefore, contracture did not recur and a follow-up operation was not necessary. Since skin grafts were deeply placed in fossa (aside the flap), the grafts were barely noticeable. With time, skin grafts accepted normal properties (color, thickness, elasticity) very closely resembling the skin of lateral phalanges' surfaces; thus, the split skin grafts did not present cosmetic or neurovascular problems. The trapezoid adipose-cutaneous flaps did no shrink; being stretched, the flaps' and surrounding stretched skin continued to grow, restoring and surpassing initial flaps' width which prevented contracture recurrence in adults and more importantly in pediatric patients. As a result of flap growth, the tissue stretching normalized as well as the commissural space and interdigital fossa's depth. In case of dorsal hand resurfacing, the trapezoid flap formed a normal commissural groove and slant. With time, the follow-up results improved: as operation scars 


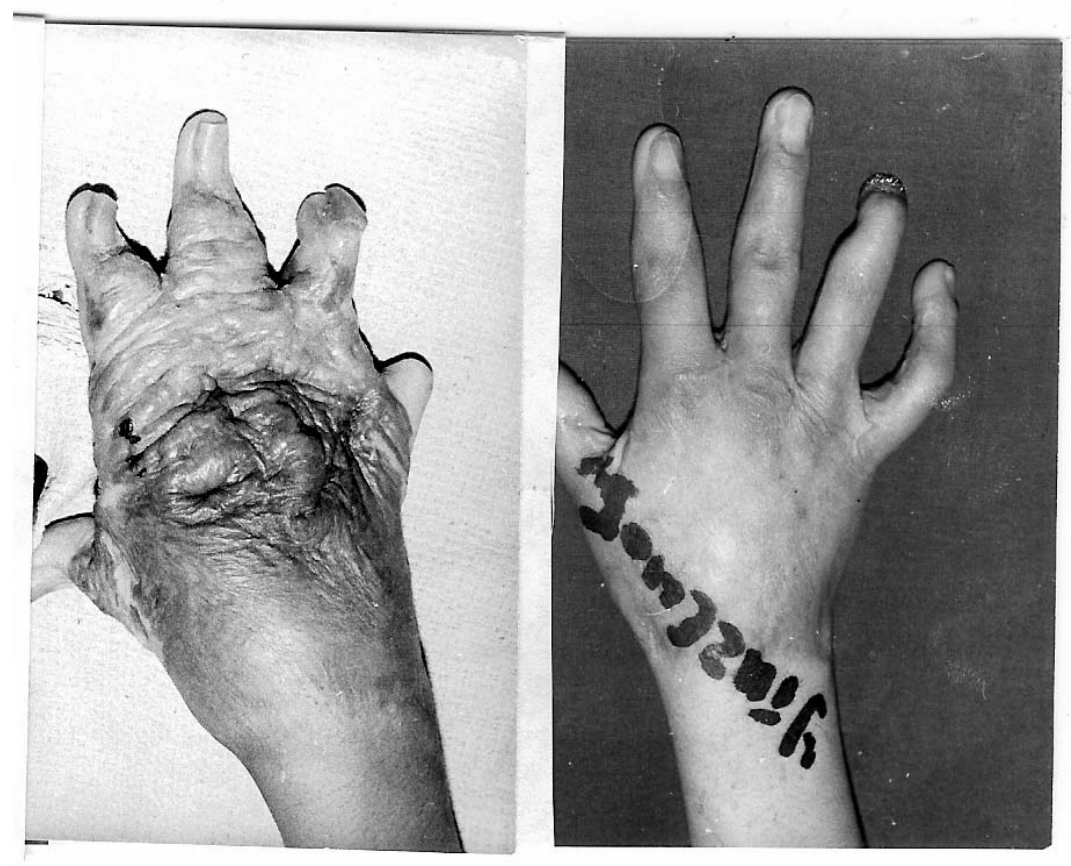

(a)

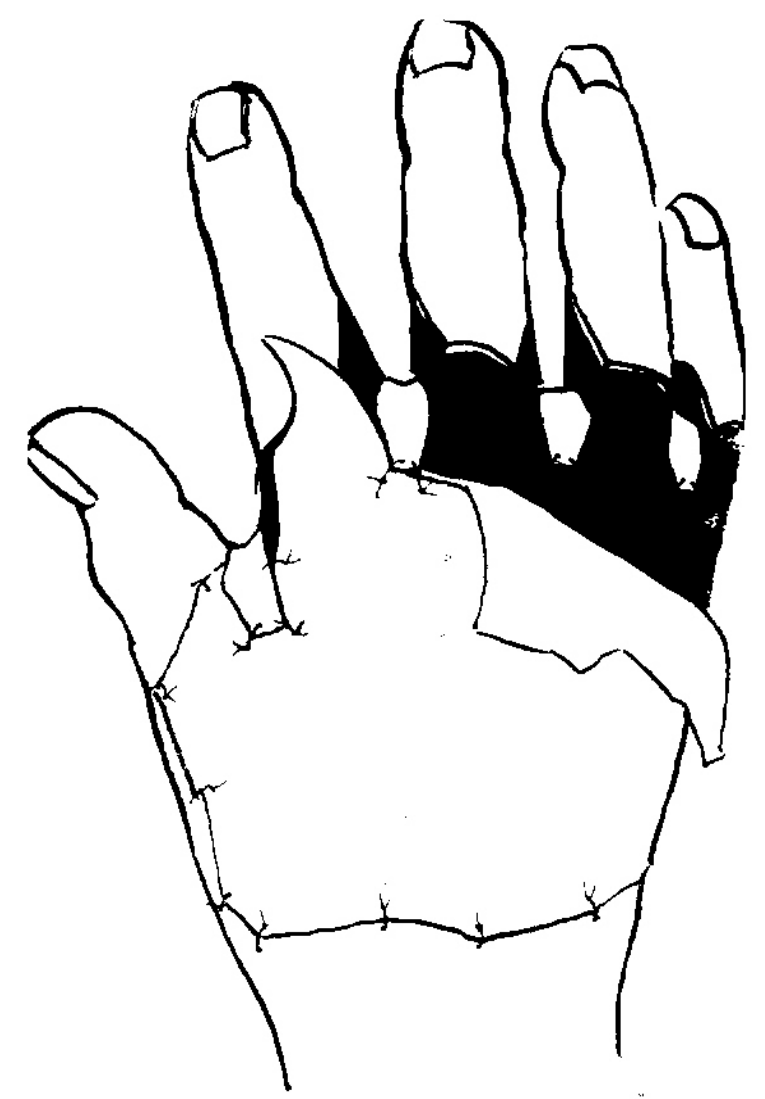

(b)

Figure 4. Technique of commissure restoration in case of total dorsal hand resurfacing. A, upper-prior to surgery; (lower)-result of operation: contracture released, groove and slant fully restored, dorsal hand surface resurfaced; B-scheme of operation. 
and grafts matured, flaps became bigger, fully restoring interdigital fossa, commissural groove and slant.

\section{DISCUSSION}

First of all, it is important to note that commissural postburn scar contractures differ from the congenital syndactyly anatomically; therefore, the reconstructive techniques and criteria for evaluating the results are also different. An adequate reconstructive technique should fully release the commissural contracture, fully restore the interdigital fossa, groove, and slant. Based on these criteria, one can evaluate the effectiveness of every curative procedure. Many techniques and flaps have been previously proposed and used for burned commissure reconstruction: Z-plasty, multiple Z-plasty techniques, skin grafting [58]. Parry reported [9] that Z-plasty gives optimal results. According to German and Phillip [10], the technique of simple Z-plasty gives the greatest increase in length and depth of the web space. Mlakar and Dougherty [11] suggest that dorsal hooding can be released with multiple zplasties, five-flap z-plasties or a series of releases and grafting; in cases of significant burn syndactyly, the dorsal pedicle flap from the remaining soft tissues on the digits can be elevated to interpose within the base of the web. Kurtzman and Stern [11] reported that local flap coverage may be adequate in mild contractures, but moderate-to-severe injuries will require a combination of skin grafting with or without local wascularized tissue.

Other flaps and techniques that had been recommended are as follows: five-flap, $\mathrm{Y}-\mathrm{V}$ advancement with Z-plasty [12]; reverse triangular V-Y flaps, using two reverse $\mathrm{V}-\mathrm{Y}$ island triangular flaps [13]; five-flap technique which combines a double opposing Z-plasty and with a Y-V plasty [14]; spearhead procedure (derived from V-Y plasty and the hourglass flap), making use of a local skin flap, drawn on the dorsal aspect of the hand [15]; V-M plasty and its modification (considered a good choice for release and reconstruction of postburn syndactyly and carried out with little complications) [16-18]. Sood et al. [7] considers V-M plasty one of the preferred methods; V-N plasty has been noted [19]; three-squareflip-flap method is considered a simple, reliable, and safe method for treatment of postburn syndactyly [20]; dorsal rectangular scar flap [21]; four flaps' technique [22]; STAR-plasty, use of a single volar flap, harvested from each sidewall of both proximal phalanges, with 30-degree corners; method deepens and widens the web space [23]; "Seagull" flap [24]; rectangular flap from lateral finger surface [2]; cross incision plasty combined with longitudinal and transverse release [25]; dorsal rectangular flap with a single palmar triangular flap and fullthickness skin graft for palmar syndactyly [12]; scar island band rotation flap [26]; and lateral volar finger flap [27].
An analysis of 190 operations for correction of postburn syndactyly presented by Alexander et al. [16] showed that none of the procedures commonly used was entirely satisfactory because of partial recurrence of lesions and/or less than satisfying cosmetic results. Complete correction of the dorsal slant was a particular problem.

Not all publications and flaps are listed in this section (in past three years the attention to postburn syndactyly treatment decreased), but the presence of so many flaps and techniques for reconstruction of a simple contracture testifies of their ineffectiveness. Similar picture is observed in regards to treatment of burn contractures of other locations. Reflecting on the field of burn reconstructions, Klein [28] concludes that it is apparent that one should expect an evolution in surgical techniques and technologies that can improve the function and appearance of persons with burn injuries. It becomes quite evident that: a) treatment of interdigital commissural scar contractures is tangled; b) contracture anatomic features are not researched sufficiently; c) the suggested techniques are far from perfect; thus d) it is necessary for research to continue as the problem of preferable technique choice persists.

Most of the aforementioned techniques were tested in our institute and brought neither the surgeon nor the patients desired outcomes: contractures recurred after skin grafting, commissure restoration with triangular flap plasties was incomplete, or yet another secondary hand deformity occurred after flaps' harvesting from fingers. The procedures commonly used and based on triangular local flap-plasty (including Y-V plasty and V-M technique and their modifications) are unable to completely compensate scar surface deficiency, fully release the contracture, and restore the interphalangeal groove and slant due to two reasons: the triangular flaps do not match the scar surface deficiency (trapezoid), and the flaps themselves are of insufficient length.

The results of our study laid ground for development of a new anatomic scar contracture classification that prompted the new approach for the following surgical techniques.

Scar contractures classification and terminology. Today, postburn scar contractures of the scar joints are categorized based on their function flexion, extension, or adduction and abduction. In spite of great years of medical history and research, the anatomy and nature of scar contractures has not been sufficiently studied. Thus, no classification based on anatomic data (basis for reconstructive surgery) has been presented.

After studying several thousands of scar contractures, we determined that scar contracture formations follow a certain set of strict physiological principles [29]. Formation type depends on the joint's or commissural side injured 

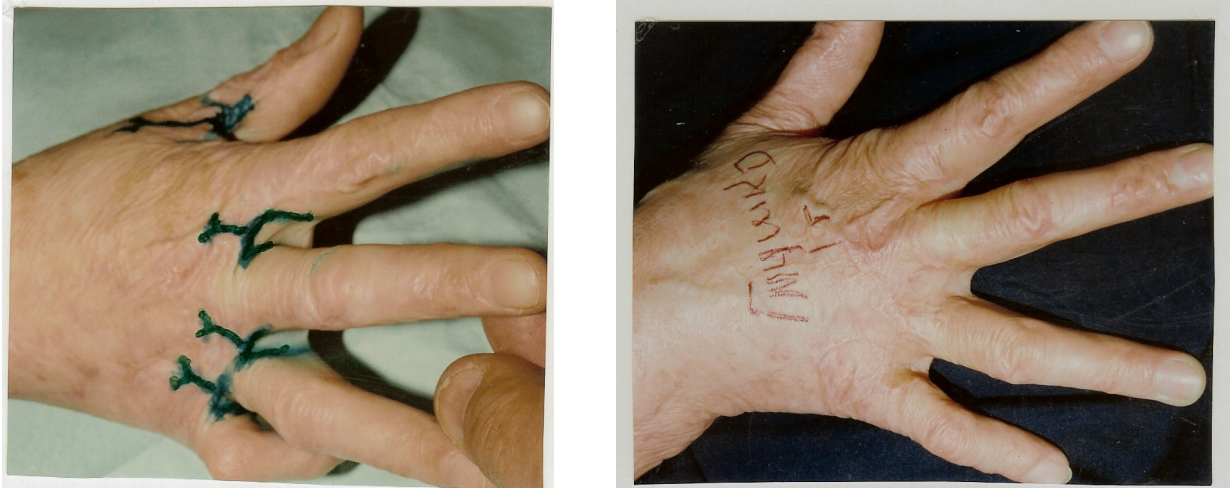

(a)
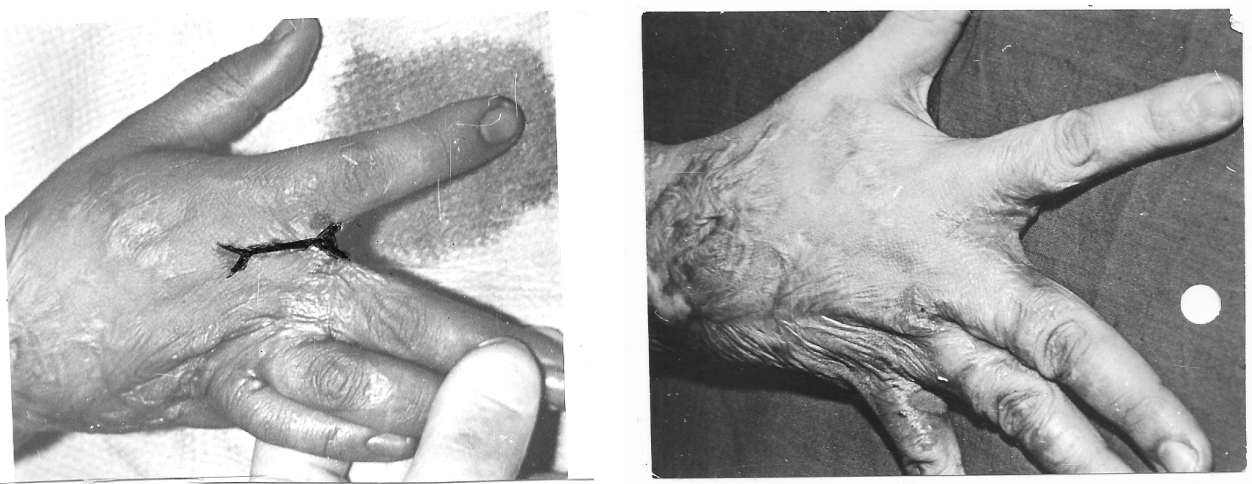

(b)
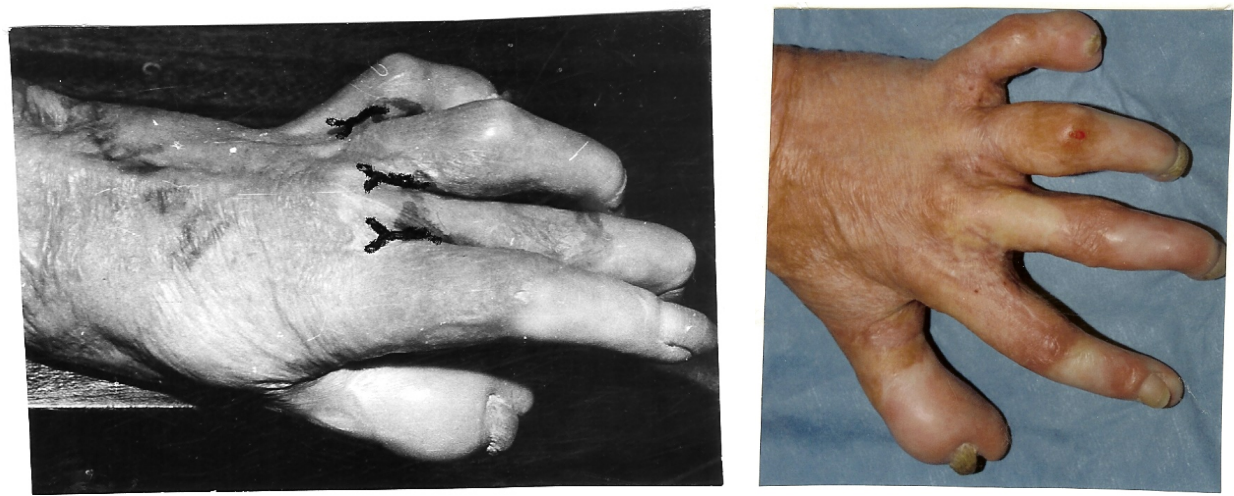

(c)
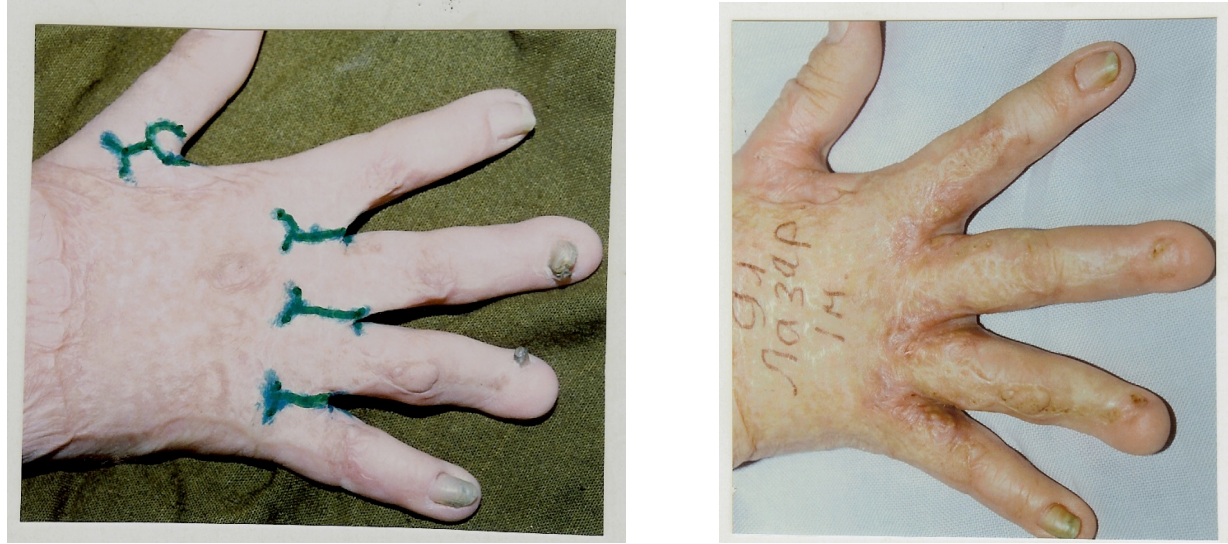

(d) 

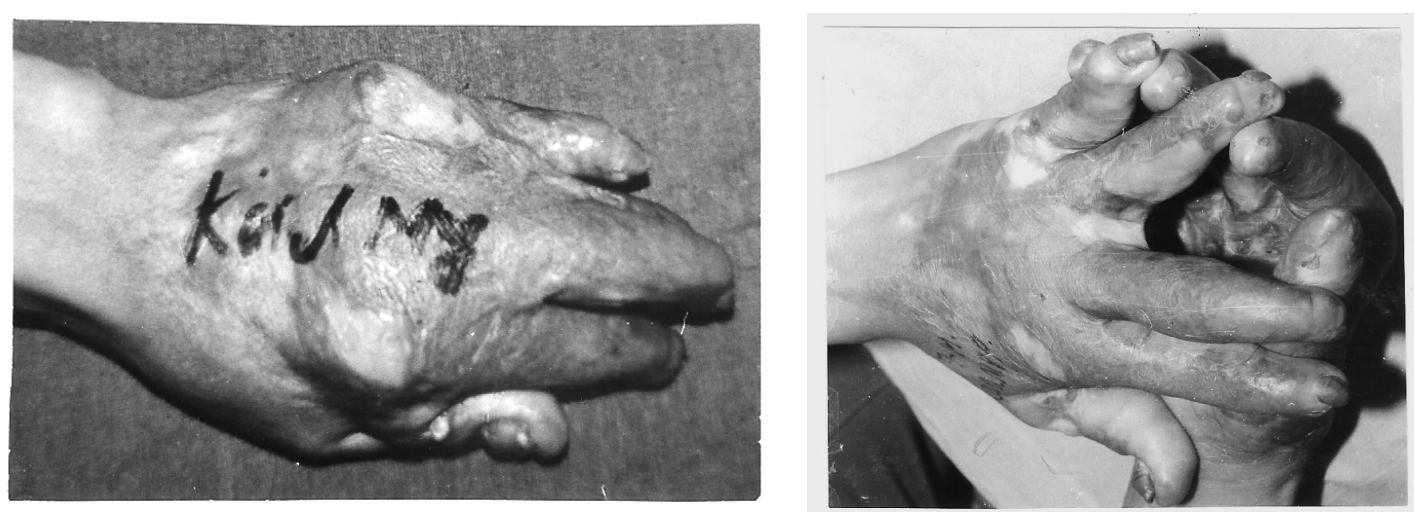

(e)

Figure 5. Follow-up results of postburn edge dorsal commissural contractures elimination with trapeze-flap plasty: scar surface deficit (contracture cause) compensated by flap, interdigital fossa, interphalangeal groove, and slant restored.

and covered with scars: flexion, lateral or all three joint's sides (in relation to the joint), or lateral or inner surface of commissure (interdigital, oral, eye's). All postburn scar contracture were classified into three types: edge, medial, and total.

Edge contractures. Contracted scars, located on the lateral commissural or joint's surface, form a fold, located along the commissural edge (dorsal or palmar interphalangeal, lateral oral, or eye) or flexion joint's surface (or fossa edge-cubital, axillary, and popliteal). The fold consists of two sheets. The lateral (according to commissural or joint's fossa) is scars, and it causes the contracture due to scar surface deficiency in the fold's length. The medial sheet of the fold and the adjacent fossa's is healthy, allowing contracture elimination with local and adjacent tissues. The crest of the fold is the edge of scars. In other words, a contracture that is classified as edge must possess the following features: a) caused by the fold; b) the fold is located along the commissural or joint's surface edge; c) the fold has a crest which is the edge of scars, and d) only one sheet is scars, and other sheet is healthy tissue. These specific anatomic criteria are observed regardless of contracture location. Dorsal and palmar interdigital scar contractures belong to the edge type.

Medial contractures. The contracture caused by the scar fold can be classified as medial when strictly fulfilling the following anatomic criteria: a) the fold is located along the medial commissural or joint's flexion surface line; b) both sheets of the fold are scars. In the scar sheets, there is surface deficiency in fold's length (cause of contracture) and scar surface surplus in width (from the fold's crest to the joint rotation axis) which allows contracture elimination with local tissues. If responding to the above-mentioned criteria, the contracture type becomes medial regardless of contracture location. The medial contracture does not occur in $2^{\text {nd }}-4^{\text {th }}$ interdigital spaces.

Total contractures. Postburn contracture, caused by scars, obliterating commissural fossa or tightly surrounding the joint on all three sides-flexion and both lateral without fold-is classified as total. Total contracture type is characterized by severe scar surface deficiency, excluding any application of local-flap plasty. The new scar contracture classification advanced a new, scientifically justified terminology [29].

The key to solving of the treatment problem lies in understanding of the commissural digital contracture anatomy, mainly realizing that the contracture is of the edge type 1) The contracture is caused by the fold located along the anterior or posterior fossa's edge; only the lateral fold's sheet is scars and it causes the contracture itself; 2) in the scar sheet of the fold there is a scar surface deficiency which has a trapezoid shape and spreads from the fold's crest to the metacarpal bone heads; 3) the medial sheet (in relation to interdigital fossa) and interdigital fossa's skin is healthy. Dorsal hand scars not only form the contracture, but also cause the interphalangeal groove smoothening and the slant's disfigurement by the fold. Therefore, reconstruction should include: a) complete contracture release up to metacarpal bone heads' level; b) scar surface deficiency (wound)/projection, and adequate compensation (wound resurfacing) with a similarly shaped (trapezoid) flap leading to the interphalangeal groove and slant restoration.

The flaps, created by Z- and Y-V plasties and their multiple modifications, are triangular and, therefore, do not correspond to the wound's (scar surface deficiency) form which is, as a rule, a trapezoid. Thus, the flaps with acute angles are insufficient for an adequate contracture elimination and groove and slant restoration. Moreover, the triangular pointed flaps are often not mobilized due to necrosis danger; therefore, their displacement is also restricted and insufficient in length to reach the metacar- 
pal bone heads which is necessary for groove and slant restoration. Thus, the wound (scar surface deficiency) shape predetermines the flap's form, not the other way around.

Consequently, the challenge of the reconstruction is to find the donor site from which one could raise a similarly-shaped (trapezoid, long), healthy-skin flap with minimal chances of donor site morbidity. Our optimal resolution was using the undamaged interdigital fossa and adjacent lateral surfaces of proximal phalanges for flap construction of necessary size and shape. The flaps have a wide end which forms the fossa between metacarpal bone heads. It does not undergo rotation and has steady blood circulation which ensures an uncomplicated postoperative course. The donor wounds on lateral surfaces of proximal phalanges are small and deeply located; therefore, the skin transplants are hardly visible and present no cosmetic discomfort.

This new approach is based on new anatomic data (scar surface deficiency, its trapezoid form and spreading to the metacarpal bone heads) and on a new reconstructive technique (trapeze-flap plasty) which allows full interdigital scar contracture elimination and interphalangeal groove and slant restoration. Within a short period of time, the trapeze-flap plasty became the main surgical procedure and superseded all other previously used techniques. There were no contraindications for trapeze-flap plasty if the skin of interdigital fossa was not injured.

Trapeze-flap plasty possesses numerous advantages: a) the trapezoid adipose-cutaneous flap corresponds with the scar surface deficiency; therefore, the deficiency is compensated and the contracture is eliminated in full; b) the flap restores the commissural groove and slant; c) the flap has steady blood circulation and does not undergo rotation; thus, its transposition with tension up to the metacarpophalangeal joint is not dangerous; d) the flap is elevated from the bottom of interdigital space (fossa) which remained to be covered with the same flap; e) the extended flap continued to grow, normalizing the interdigital fossa's depth, preventing the contracture recurrence which is especially important for pediatric patients; f) small transplants, located deeper on the lateral surface of proximal phalangs, do not pose a cosmetic defect and the donor site morbidity is minimal. The technique is easy to plan and perform. There are no contraindications for the application of this method. There is no need for immobilization or occupational therapy after surgery. Introduction of the trapeze-flap plasty simplified the procedure of burned commissural contractures reconstruction making it the easiest and most effective technique most often used for burned hand reconstruction.

\section{CONCLUSION}

Dorsal and palmar interdigital scar commissural con- tractures can be identified as edge contractures that are caused by the fold located along the interdigital fossa's edge, where interphalangeal groove is smoothed and slant is disfigured. The contracted scars have trapezeshaped surface deficiency (contracture cause), spreading from the fold's crest to the metacarpal phalangeal heads. Reconstruction includes scar surface deficiency compensation using original approach, based on adipose-cutaneous trapezoid flap, mobilized in interdigital fossa and transposed on the dorsal hand. Small donor wounds on the lateral proximal phalanges' surfaces are grafted. Complete contracture elimination, commissural anatomy restoration, and good cosmetic outcomes make the trapezeflap plasty procedure the one of choice. The author believes that once one uses this technique, he will resort to no other.

\section{REFERENCES}

[1] Kalliainen, L.K. and Schubert, W. (2005) The management of web space contractures. Clinical Plastical Surgery, 32, 503-514. doi:10.1016/j.cps.2005.06.002

[2] Gulgonen, A. and Ozer, K. (2007) The correction of postburn contractures of the second through fourth web space. Journal of Hand Surgery, 32A, 556-564. doi:10.1016/j.jhsa.2007.01.017

[3] Grishkevich, V.M. (2010) Trapeze-flap plasty: An effective technique for postburn edge elbow contracture elimination. Shoulder and Elbow, 2, 273-280. doi:10.1111/j.1758-5740.2010.00077.x

[4] Grishkevich, V.M. (2011) Post-burn microstomia: Anatomy and elimination with trapeze-flap plasty. Burns, 37, 484-489. doi:10.1016/j.burns.2010.09.003

[5] Smith, M.A., Munster, A.M. and Spence, R.J. (1998) Burns of the hand and upper limb-A review. Burns, 24, 493505. doi:10.1016/S0305-4179(98)00063-1

[6] Asuku, M.E., MacCauley, R.L. and Piazza, R.C. (2005) Reconstruction of the burned hand. In: MacCauley, R.L., Ed., Functional and Aesthetic Reconstruction of Burned Patients, Taylor and Francis, Boca Raton, 437-462.

[7] Sood, R., Brenner, K., Bruce, M. and Achauer, B.M. (2006) Reconstruction of the burned hand. In: Sood, R., Ed., Achauer and Sood's Burn Surgery. Reconstruction and Rehabilitation, Saunders, Elsevier, 307-323.

[8] Parry, S.W. (1989) Reconstruction of the burned hand. Clinical Plastical Surgery, 16, 577-586.

[9] Germann, G. and Phillip, K. (2005) The burned hand. In: Green, D.P., Hotchkiss, R.N., Pederson, W.C., Wolfe, S.W., Eds., Green's Operative Hand Surgery, Elsevier Churchill Livingstone, Philadelphia, 2159-2190.

[10] Mlakar, J.M. and Dougherty, W.R. (2002) Reconstruction of the burned hand. In: Herndon, Ed., Total Burn Care, W.B. Saunders, Philadelphia, 628-655.

[11] Kurtzman, L.C. and Stern, P.J. (1990) Upper extremity burn contractures. Hand Clinics, 6, 261-279.

[12] El-Din, S.A.S., El-Din, A.B., El-Shafeey, E.-S. and Shou- 
man, O.M. (2000) Reconstruction of post burn palmar web contractures: A surgical approach. Egypt Journal of Plastic and Reconstructive Surgery, 23, 29-33.

[13] Savaci, N., Hosnuter, M. and Tosun, Z. (1999) Use of reverse triangular $\mathrm{V}-\mathrm{Y}$ flaps to create a web space in syndactyly. Annals of Plastic Surgery, 42, 540-544. doi:10.1097/00000637-199905000-00013

[14] Rousso, M. and Wexler, R.M. (1978) Secondary reconstruction of the burned hand. Progressive Surgery, 16, 182-206.

[15] Rouge, D., Bondar, R., Grolleau, J.L., Braye, F., Micheau, P., Chavoins, J.P. and Costagliola, M. (1996) Spearhead procedure for repair of web space burn sequelae. European Journal of Plastic Surgery, 19, 77-80. doi:10.1007/BF00207919

[16] Alexander, J.W., MacMillan, B.G. and Martel, L. (1982) Correction of postburn syndactyly: An analysis of children with introduction of the WM plasty and postoperative pressure insert. Plastic and Reconstructive Surgery, 70, 345-354. doi:10.1097/00006534-198209000-00009

[17] Davami, B. (2009) V-M plasty and double Z-plasty: Two versatile flaps for treatment of postburn syndactyly. Techniques in Hand \& Upper Extremity Surgery, 13, 124-129. doi:10.1097/BTH.0b013e3181a6b5e1

[18] Tan, O., Atik, B. and Ergen, D. (2005) Versatile use of the VM-plasty for reconstruction of the web space. Annals of Plastic Surgery, 55, 623-628. doi:10.1097/01.sap.0000181651.66907.8f

[19] El Kollali, R., Ghoneim, I. and Al-Azemi, M. (2006) V-N plasty for release of severe postburn contractures. Journal of Plastic, Reconstructive \& Aesthetic, 59, 1424-1428. doi:10.1016/j.bjps.2006.01.043

[20] Lapid, O. and Sagi, A. (2005) Three-square-flip flap reconstruction for post burn syndactyly. British Journal of Plastic Surgery, 58, 826-829. doi:10.1016/j.bjps.2005.04.001
[21] Browne Jr., E.Z. Teague, M.A. and Snyder, C.C. (1972) Burn syndactyly. Plastic and Reconstructive Surgery, 62, 92-95. doi:10.1097/00006534-197807000-00014

[22] Rampazzo, A., Gharb, B.B. and Bassetto, F. (2008) "Four flap's technique" for the reconstruction of grade 4 postburn dorsal neosyndactyly. Burns, 34, 144-147. doi:10.1016/j.burns.2007.04.015

[23] Hultman, C.S., Teotia, S., Calvert, C., Thorntton, S. and Schram, J. (2005) STAR plasty for reconstruction of the burned web space: Introduction of an alternative technique for the correction of dorsal neosyndactyly. Annals of Plastic Surgery, 54, 281-287.

[24] Smith, P.J. and Harrison, S.H. (1982) The "seagull” flap for syndactyly. British Journal of Plastic Surgery, 35, 390393.

[25] Emsen, I.M. (2008) The cross incision plasty for reconstruction of the burned web space: Introduction of an alternative technique for the correction of dorsal and volar neosyndactyly. Journal of Burn Care and Research, 29, 378-385. doi:10.1097/BCR.0b013e31816673cd

[26] Murakami, M., Hyakusoku, H. and Ogawa, R. (2005) Band rotation flap. Burns, 31, 220-222. doi:10.1016/j.burns.2004.09.012

[27] MacDougal, B., Wray, R.C. and Weeks, P.M. (1976) Lateral-volar finger flap for the treatment of burn syndictyly. Plastic and Reconstructive Surgery, 57, 167-712. doi:10.1097/00006534-197602000-00006

[28] Klein, M.B. (2011) Burn reconstruction. Physical Medicine and Rehabilitation Clinics of North America, 22, 311-326. doi:10.1016/j.pmr.2011.01.002

[29] Grishkevich, V.M. (1991) The basic types of scar contractures after burns and methods of elimination them with trapeze plasty flaps. Plastic and Reconstructive Surgery, 88, 1044-1054. doi:10.1097/00006534-199112000-00015 